\title{
Acute effects on human sperm exposed in vitro to cadmium chloride and diisobutyl phthalate
}

\author{
S Marchiani ${ }^{1, *}$, L Tamburrino ${ }^{2, *}$, G Farnetani ${ }^{2}$, M Muratori $^{3}$, L Vignozzi $^{3}$ and E Baldi ${ }^{2}$ \\ ${ }^{1}$ Azienda Ospedaliero Universitaria Careggi, Florence, Italy, ${ }^{2}$ Department of Experimental and Clinical Medicine, \\ University of Florence, Florence, Italy and ${ }^{3}$ Department of Experimental and Clinical Biomedical Sciences 'Mario \\ Serio', Center of Excellence DeNothe, University of Florence, Florence, Italy
}

Correspondence should be addressed to S Marchiani; Email: sara.marchiani@unifi.it

*(S Marchiani and L Tamburrino contributed equally to this work)

\begin{abstract}
Epidemiological studies reported a negative relationship between concentrations of heavy metals and phthalates in seminal fluid and semen quality, likely compromising male fertility potential. The aim of this study was to investigate the in vitro effects of cadmium chloride $\left(\mathrm{CdCl}_{2}\right)$, a common heavy metal, and diisobutyl phthalate (DIBP), a common phthalate ester, on human sperm functions necessary for fertilization. After in vitro incubation of spermatozoa with $10 \mu \mathrm{M} \mathrm{CdCl}_{2}$ or 100 and $200 \mu \mathrm{M} \mathrm{DIBP}$ for $24 \mathrm{~h}$, a significant decrease of sperm progressive and hyperactivated motility was observed. The exposure to each of the two toxic agents also induced spontaneous sperm acrosome reaction and blunted the physiological response to progesterone. Both agents induced an increase of caspase activity suggesting triggering of an apoptotic pathway. Our results suggest that acute exposure of spermatozoa to these pollutants may impair sperm ability to reach and fertilize the oocyte.

Reproduction (2019) 158 281-290
\end{abstract}

\section{Introduction}

Biomonitoring studies indicate that the levels of environmental contaminants rose substantially in last 3-4 decades exposing the population to toxicity of such compounds, which tend to accumulate in the organism and to induce multiple organ alterations. These exogenous agents are represented by a heterogeneous group of substances, normally present in the environment (air, water, soil etc.) but also in mass-consumer products and food, which can behave as endocrine disruptors interfering with normal hormonal activity. Among the endocrine disruptors, heavy metals, bisphenol $A$, phthalates, parabens and pesticides can be found in biological fluids at various concentrations. Epidemiological studies demonstrated that such chemicals can impact on human health disrupting the normal function of the endocrine system, and subsequently, produce disorders at developmental, neurological, cardiovascular, metabolic, immune and also reproductive level (Wang et al. 2016a, 2019). Indeed, environmental and occupational exposure of the population could be one of the risk factors of the increased incidence of male infertility and the decline of human semen quality seen in the last decades (Levine et al. 2017, Mínguez-Alarcón et al. 2018). In general, infertility affects $15 \%$ of the couples and the male factor contributes for about $50 \%$ of them. Besides couple infertility, a decline of male reproductive health could have wider public health implications, as the deterioration of the semen quality is associated to increased morbidity and mortality (Jensen et al. 2009, Eisenberg et al. 2014, 2016, Latif et al. 2017).

Recent epidemiological evidence reported increased risk of reproductive disorders following prenatal and postnatal persistent exposure to environmental chemicals (Bonde et al. 2016, Wang et al. 2016a, Sifakis et al. 2017). For example, alterations of semen parameters have been reported following exposure to phthalates, commonly used as plasticizers (Radke et al. 2018) and in subjects with high semen concentrations of heavy metals, widely used in industry (Sun et al. 2017). In addition, high levels of heavy metals in follicular fluid and high concentrations of phthalate metabolites in female urine decrease assisted reproduction outcomes (Wdowiak et al. 2017, Al-Saleh et al. 2019).

Data from animal and epidemiological studies on the effects of toxicants on male reproductive health are still insufficient and conflicting and the few experimental studies on human beings do not allow establishing definite evidence (Tavares et al. 2016, de Angelis et al. 2017). Pant et al. (2014) found that seminal levels of cadmium and phthalate esters are negatively associated with sperm motility and concentration and positively 
related with DNA damage in non-occupationally exposed subjects. Also, in animal models, reduced plasma and testis testosterone levels, steroidogenic enzyme expression and semen quality, have been shown after exposure of adult male mice to diisobutyl phthalate (Pan et al. 2017) as well as testis toxicity and morphological and functional alterations of spermatozoa following cadmium administration in rats (Medina et al. 2017). However, considering that spermatozoa must 'travel' in the female genital tract where they undergo selection as well as profound modifications of motility (development of hyperactivated motility necessary to penetrate oocyte vestments) and acrosome reaction in response to physiological stimuli in order to fertilize the oocyte (Stival et al. 2016), it is crucial also to understand the possible effect of the environmental contaminants on these functions.

The present study was designed to investigate the acute effect of compounds representative of two categories of environmental pollutants on sperm quality and function. In particular, we analyzed the effects of in vitro exposure of human spermatozoa to cadmium chloride $\left(\mathrm{CdCl}_{2}\right)$, one of the most common heavy metal contaminants, present at high levels in batteries, computer components and tobacco smoke, and diisobutyl phthalate (DIBP), a common environmental phthalate ester, contained in plastic goods for food preservation, inks, paints and personal care products.

The effect of the two contaminants on sperm progressive and hyperactivated motility, sperm acrosome reaction and sperm intracellular calcium levels, parameters essential for a successful fertilization, were evaluated in swim-up selected spermatozoa in order to mimic the natural selection occurring in the female genital tract.

\section{Materials and methods}

\section{Chemicals}

Human tubal fluid (HTF) medium and human serum albumin (HSA) were purchased from Biocare Europe (Rome, Italy). Cadmium chloride hydrate $\left(\mathrm{CdCl}_{2}\right)$, diisobutyl phthalate (DIBP), FITC-labeled Arachis hypogea (peanut) lectin and progesterone were obtained from Sigma Aldrich. $\mathrm{CdCl}_{2}$ was dissolved in water to a stock concentration of $100 \mathrm{mM}$. DIBP original stock solution $(3.6 \mathrm{M})$ was diluted with ethanol to obtain $100 \mathrm{mM}$ working solution. The lectin was diluted $1 \mathrm{mg} /$ $\mathrm{mL}$ in phosphate-buffered saline, $\mathrm{pH}$ 7.2. Progesterone was dissolved in DMSO to obtain $0.1 \mathrm{M}$ working solution.

Fura 2/AM Cell Permeant and Vybrant FAM Caspase 3 and 7 Assay Kit were purchased from Life Technologies Italia.

\section{Human semen samples}

Semen samples were obtained by masturbation from normozoospermic patients undergoing routine semen analysis for couple infertility, in the Andrology Laboratory of the
University of Florence, Italy. The study was approved by the local ethical committee (protocol n. 8607/2018, cod. 12186/ OSS). Patients were informed about the purpose of the study and a written informed consent to use the semen remaining after routine analysis was obtained. Semen analysis was carried out according to guidelines of the World Health Organization (World Health Organization (WHO) 2010). Semen samples with leukocytes were excluded from the study, after assessment of their presence according to WHO (2010). The Laboratory of Andrology of the Azienda Ospedaliera-Universitaria Careggi of Florence has been participating in the UK-NEQAS (United Kingdom National External Quality Assessment Service) external quality control program for semen analysis since 2005. The mean ( \pm S.D.) percent biases of the laboratory for the years 2018 were $8.9( \pm 6.6)$ and $15.9( \pm 12.8)$, respectively, for total and progressive motility and $9.1( \pm 5.3)$ for sperm concentration $(n=20$, data from UK-NEQAS). For the study purpose, sperm were selected by direct swim-up procedure (WHO 2010) from 46 subjects (sperm concentration median value: $114.0 \times 10^{6} / \mathrm{mL}$ (IQR: $\left.68.8-165.5\right)$ ); progressive motility median value: $58.0 \%$ (IQR: 48.8-65.0); total motility median value: $65.0 \%$ (IQR: $58.0-71.3$ )) in order to obtain a sufficient number of selected spermatozoa to perform the experiments. Briefly, $1 \mathrm{~mL}$ of HTF $-10 \%$ HSA was gently layered on an equal volume of semen sample and incubated at $37^{\circ} \mathrm{C}$. After $50 \mathrm{~min}, 800 \mu \mathrm{L}$ of the upper medium phase, containing the motile fraction of spermatozoa, was collected. In all samples, motility was evaluated and the obtained sperm number was counted according to WHO (2010). Only those samples with a progressive motility $>90 \%$ were used for further experiments.

\section{Dose and time response experiments}

In order to choose the concentrations of $\mathrm{CdCl}_{2}$ and DIBP as well as the time of exposure to use in the following experiments, we tested the effect of increasing concentrations and incubation times of the two toxicants on sperm motility. Swim-up selected spermatozoa (10 millions) were incubated in $1 \mathrm{~mL}$ of $\mathrm{HTF}-10 \% \mathrm{HSA}$ with $\mathrm{CdCl}_{2}$ at the doses of 2, 5 and $10 \mu \mathrm{M}$ or with DIBP at the doses of 25,50, 100 and $200 \mu \mathrm{M}$ for 1,3 and $24 \mathrm{~h}$ at $37^{\circ} \mathrm{C}, 5 \% \mathrm{CO}_{2} . \mathrm{CdCl}_{2}$ concentrations were chosen on the basis of previous studies evaluating cadmium levels in seminal fluid (Pant et al. 2013) and its in vitro effect on human spermatozoa (Pant et al. 2013). DIBP concentrations were chosen on the basis of the levels of phthalates detected in semen (Pant et al. 2011) and up to the high doses within the range of phthalates concentrations tested in prior in vitro studies (Pant et al. 2011, Adir et al. 2017a,b).

For each sample a control aliquot containing HTF $-10 \%$ $\mathrm{HSA}$ and the solvent (water for $\mathrm{CdCl}_{2}$ and ethanol for DIBP) was carried out. After incubation, the percentage of progressive and total sperm motility were checked by phasecontrast microscope (Leica DMLS; Leica, Wetzlar, Germany). As shown in the Supplementary Fig. 1 (see section on supplementary data given at the end of this article), $\mathrm{CdCl}_{2}$ at the concentrations of 2 and $5 \mu \mathrm{M}$ had no statistically significant effects on sperm motility, even after a long incubation ( $24 \mathrm{~h})$, whereas $10 \mu \mathrm{M} \mathrm{CdCl}_{2}$ significantly decreased both progressive and total motility, starting from the first hour of incubation 
Table 1 Number of analyzed samples for each sperm parameter.

\begin{tabular}{|c|c|}
\hline $\begin{array}{l}\text { Number } \\
\text { of samples }\end{array}$ & Sperm parameter \\
\hline 46 & Progressive and total motility \\
\hline 33 & Computer-assisted sperm analysis \\
\hline 23 & Viability \\
\hline 7 & Caspase activity \\
\hline 10 & Spontaneous and progesterone-induced acrosome reaction \\
\hline 9 & $\begin{array}{l}\text { Spontaneous and progesterone-induced intracellular } \\
\text { calcium levels }\end{array}$ \\
\hline
\end{tabular}

(Supplementary Fig. $1 \mathrm{~A}$ and B). Regarding incubation with DIBP, no effects were observed after both 1 and $3 \mathrm{~h}$ of treatment, even at the maximum dose $(200 \mu \mathrm{M})$. After $24 \mathrm{~h}$, the concentration of $100 \mu \mathrm{M}$ DIBP induced a notable reduction of progressive motility and a slight decrease of total motility (Supplementary Fig. 1C and D). At the concentration of $200 \mu \mathrm{M}$ the effect of DIBP on both total and progressive motility was considerably more pronounced (Supplementary Fig. 1C and D).

In light of these results, we decided to perform the subsequent experiments using the doses of $10 \mu \mathrm{M}$ for $\mathrm{CdCl}_{2}$ and $100 \mu \mathrm{M}$ and $200 \mu \mathrm{M}$ for the DIBP and the incubation time of $24 \mathrm{~h}$ (the number of analyzed samples for each sperm parameter is reported in Table 1).

\section{Assessment of sperm motility}

Sperm motility was measured by evaluating 200 spermatozoa under a phase-contrast microscope (Leica DM LS; Leica) and graded according to WHO (2010) criteria. Sperm kinematic motility parameters were determined on motile spermatozoa by using the CASA system (Computer-Assisted Sperm Analysis, Hamilton Thorn Research, Beverly, MA, USA). The settings used during CASA procedures were: analysis duration of $1 \mathrm{~s}$ (30 frames); minimum contrast, 80; minimum size, 3; low size and high size gates, 0.7 and 2.6; low-intensity and highintensity gates, 0.34 and 1.40 (Luconi et al. 2004). Average path velocity $(\mathrm{VAP}, \mu \mathrm{m} / \mathrm{s})$, straight line velocity $(\mathrm{VSL}, \mu \mathrm{m} / \mathrm{s})$, curvilinear velocity $(\mathrm{VCL}, \mu \mathrm{m} / \mathrm{s})$, amplitude of lateral head displacement $(\mathrm{ALH}, \mu \mathrm{m})$, beat cross frequency $(\mathrm{BCF}, \mathrm{Hz})$, straightness (STR, \%) and linearity of progression (LIN, \%) were recorded. A sort fraction representing the percentage of hyperactivated spermatozoa ( $\mathrm{HA}, \%)$ was identified setting manually the following threshold values: $\mathrm{VCL} \geq 150 \mu \mathrm{m} / \mathrm{s}, \mathrm{ALH}$ $\geq 7 \mu \mathrm{m}$ and LIN $\leq 50 \%$ (Mortimer et al. 1998). A minimum of 200 motile cells and 5 fields were analyzed for each aliquot. All analyses were performed at $37^{\circ} \mathrm{C}$.

\section{Assessment of sperm viability}

Sperm viability was evaluated by using eosin-nigrosin staining (WHO 2010). Sperm suspension and eosin-nigrosin stain (1:1) were mixed, incubated $30 \mathrm{~s}$ at room temperature, and then smeared on the slide. Spermatozoa were evaluated by optical microscopy using $100 \times$ oil immersion objective. A total of 200 spermatozoa for each aliquot were evaluated distinguishing between viable (unstained) or dead (stained with eosin) spermatozoa.

\section{Assessment of 3, 7 caspases activity}

Caspases activity was evaluated using Vybrant FAM Caspase-3 and -7 Assay Kit based on a fluorescent inhibitor of caspases (FLICA ${ }^{\text {TM }}$ ) (Marchiani et al. 2014).

After incubation with the two toxicants (see above), each sample $\left(4 \times 10^{6}\right.$ spermatozoa) was split into two aliquots, one was resuspended in $300 \mu \mathrm{L}$ of HTF medium and $10 \mu \mathrm{L}$ of $30 \times$ FLICA working solution was added (test sample) and the other was incubated only with HTF medium (negative control). After $1 \mathrm{~h}$ of incubation at $37^{\circ} \mathrm{C}$, samples were washed with Wash Buffer $1 \times$ and fixed with $40 \mu \mathrm{L}$ of $10 \%$ formaldehyde for $10 \mathrm{~min}$ at room temperature. Wash and fixative solutions were supplied by the kit. Sperm samples were washed again twice and resuspended in $400 \mu \mathrm{L}$ of Wash Buffer $1 \times$ containing $6 \mu \mathrm{L}$ of propidium iodide solution (PI, $50 \mu \mathrm{g} / \mathrm{mL}$ in PBS).

Samples were acquired by a FACScan flow cytometer equipped with a 15-mW argon-ion laser for excitation. Green fluorescence of caspases and red fluorescence of PI were revealed by the FL-1 (515-555-nm wavelength band) and FL-2 (563-607-nm wavelength band) detector respectively. Nucleated events in the gate (i.e. the events labeled with $\mathrm{PI}, n=8000$ ) of the characteristic forward scatter/side scatter region of sperm cells were acquired (Muratori et al. 2008). A marker was established in the dot plot of fluorescence distribution of the negative sample, including 99\% of total events. This marker was translated in the corresponding test sample and all the events beyond the marker were considered positive for caspase activity. CellQuest-Pro software program (Becton-Dickinson) was used for acquisition and analysis.

\section{Assessment of acrosome reaction}

Acrosome reaction was evaluated by fluorescent microscopy as previously reported (Krausz et al. 1995, 1996). Briefly, both control and treated samples were divided into two aliquots. An aliquot was incubated with progesterone $(10 \mu \mathrm{M})$ and the other with $0.1 \%$ DMSO (vehicle control), for $1 \mathrm{~h}$ at $37^{\circ} \mathrm{C}$. Afterward, spermatozoa were washed by centrifugation and resuspended in $500 \mu \mathrm{L}$ of hypo-osmotic swelling medium in order to evaluate acrosome reaction only in live spermatozoa. After $1 \mathrm{~h}$ at $37^{\circ} \mathrm{C}$, spermatozoa were washed again and fixed in $50 \mu \mathrm{L}$ ice-cold methanol. The sperm were layered on a slide, air-dried and stored at $-20^{\circ} \mathrm{C}$. For acrosome staining, sperm were incubated for $20 \mathrm{~min}$, in the dark, with FITC-labeled Arachis hypogaea (peanut) lectin and green fluorescence was observed under an Axiolab A1 FL fluorescence microscope (Carl Zeiss, Jena, Germany), equipped with filter set 49 and an oil immersion $100 \times$ magnification objective. For each condition, 200 curledtail (viable) cells were analyzed for their acrosomal status. For each different experimental conditions, the percentage of acrosome-reacted spermatozoa was reported.

\section{Evaluation of intracellular calcium levels}

Intracellular calcium levels were evaluated by a spectrofluorimetric method as previously reported (Luconi et al. 1999). Briefly, $10 \times 10^{6}$ spermatozoa were incubated with the Fura2/AM probe for $45 \mathrm{~min}$ at $37^{\circ} \mathrm{C}$. Then, samples were 
washed by centrifugation and incubated in $1 \mathrm{~mL}$ FM buffer (125 mM NaCl, $10 \mathrm{mM} \mathrm{KCl}, 2.5 \mathrm{mM} \mathrm{CaCl}_{2}, 0.25 \mathrm{mM} \mathrm{MgCl}_{2}$, $19 \mathrm{mM}$ sodium lactate, $2.5 \mathrm{mM}$ sodium pyruvate, $20 \mathrm{mM}$ $\mathrm{HEPES} / \mathrm{NaOH}$ ) for $30 \mathrm{~min}$ at $37^{\circ} \mathrm{C}$. Samples were washed and resuspended again in $1 \mathrm{~mL}$ FM buffer. Spermatozoa were stimulated with progesterone $(10 \mu \mathrm{M})$ which was added directly in the cuvette. $\left[\mathrm{Ca}^{2+}\right]_{i}$ was measured by using a spectrofluorimetric method with a Perkin-Elmer LS50B instrument equipped with a fast rotary filter shuttle for alternate 340 and $380 \mathrm{~nm}$ excitation. Fluorescence measurements were converted to $\left[\mathrm{Ca}^{2+}\right]_{\mathrm{i}}$ by determining maximal fluorescence with digitonin $(0.01 \%(\mathrm{w} / \mathrm{v})$ final concentration) followed by minimal fluorescence with $10 \mathrm{mM}$ EGTA, pH 10. $\left[\mathrm{Ca}^{2+}\right]_{\mathrm{i}}$ was calculated according to Grynkiewicz et al. (1985) assuming a dissociation constant of Fura 2 for calcium of $224 \mathrm{nM}$. Three determinations were made for each sample and the mean value was reported.

\section{Statistical analysis}

Statistical analysis was performed using the Statistical package for the Social Sciences version 25.0 (SPSS) for Windows. The Kolmogorov-Smirnov test was used to test the data distribution. Data are expressed as mean ( \pm S.D.) when normally distributed and as median (interquartiles, IQR) when non-normally distributed. Since the two solvents showed similar values for each of the measured parameters, they were averaged in the figures. For normally distributed parameters, differences between groups were evaluated by paired two-sided Student's t-test, whereas, for non-normally distributed parameters, the Wilcoxon signed-rank test was performed. A $P$ value of 0.05 was considered significant.

\section{Results}

\section{$\mathrm{CdCl}_{2}$ and DIBP effects on sperm motility and viability}

On the basis of preliminary results (Supplementary Fig. 1), the doses of $10 \mu \mathrm{M}$ for $\mathrm{CdCl}_{2}$ and of 100 and $200 \mu \mathrm{M}$ for DIBP, and the incubation time of $24 \mathrm{~h}$, were chosen to perform the subsequent experiments. Progressive (Fig. 1A) and total (Fig. 1B) sperm motility, evaluated on 38 samples, decreased significantly after exposure to $\mathrm{CdCl}_{2}$ and both DIBP concentrations. The percentage decrease of progressive motility was 30.0 (IQR: 17.0-42.4), 12.7 (IQR: 3.6-18.1] and 27.1 (IQR: 14.8-41.8) after incubation with $10 \mu \mathrm{M} \mathrm{CdCl}_{2}, 100$ and $200 \mu \mathrm{M}$ DIBP respectively, whereas the percentage decrease of total motility was 14.3 (IQR: 6.5-31.3) in $10 \mu \mathrm{M} \mathrm{CdCl}_{2}$-treated samples and 7.3 (IQR: 0.9-12.3) and 20.3 (IQR: 12.7-36.6) in those treated with $100 \mu \mathrm{M}$ and $200 \mu \mathrm{M}$ DIBP. Although not statistically significant, an increase of the percentage of non-progressive motile spermatozoa after exposure to both pollutants was also observed (median value of non-progressive motility: 5.0\% (IQR: 2.0-7.3) in CTRL samples, $8.0 \%$ (IQR: 5.0-10.0) in $10 \mu \mathrm{M} \mathrm{CdCl}_{2}$-treated samples, $6.5 \%$ (IQR:
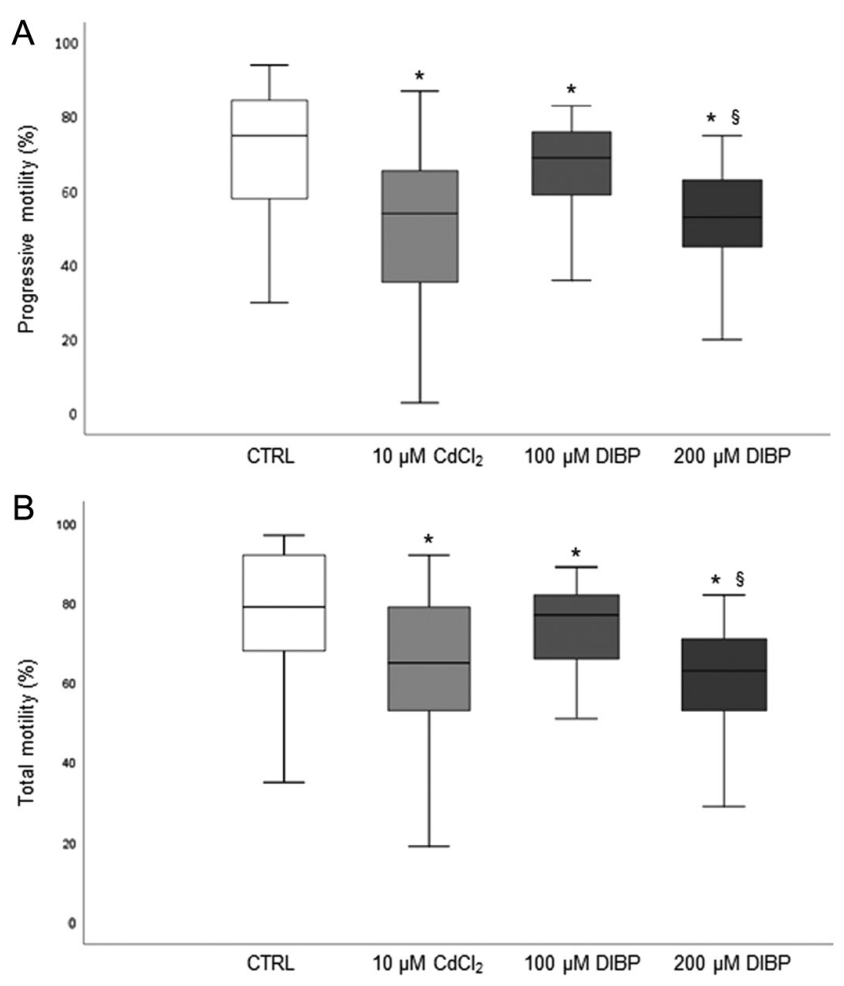

Figure 1 Box plots represent percentage of sperm progressive (A) or total (B) motility in control samples (CTRL) and in samples exposed to $10 \mu \mathrm{M} \mathrm{CdCl}_{2}$ or $100 \mu \mathrm{M}$ DIBP or $200 \mu \mathrm{M}$ DIBP for $24 \mathrm{~h}(n=38)$. Wilcoxon test: ${ }^{*} P<0.05$ vs CTRL; ${ }^{\circledR} P<0.05$ vs $100 \mu \mathrm{M}$ DIBP.

3.0-10.0) in $100 \mu \mathrm{M}$ DIBP-treated samples and $6.0 \%$ (IQR: 3.8-12.5) in $200 \mu \mathrm{M}$ DIBP-treated samples).

Most of the kinematic parameters measured by CASA system on 25 samples, underwent a significant decrease after incubation with $\mathrm{CdCl}_{2}$ and DIBP (Table 2). In particular, the percentage of hyperactivated spermatozoa was reduced by 55.0 (IQR: 22.9-71.9), 16.7 (IQR: 4.3-45.8) and 51.8 (IQR: 1.9-79.3) after incubation with $10 \mu \mathrm{M} \mathrm{CdCl}_{2}, 100$ and $200 \mu \mathrm{M}$ DIBP, respectively.

The percentage of viable spermatozoa was reduced by 6.3 (IQR, 0.0-13.2) in samples treated with $10 \mu \mathrm{M}$ $\mathrm{CdCl}_{2}$, and of 11.3 (IQR: 5.4-18.9) in samples incubated with $200 \mu \mathrm{M}$ DIBP (Fig. 2, $n=15$ ). $100 \mu \mathrm{M}$ DIBP did not affect sperm viability (Fig. 2, $n=15$ ).

\section{$\mathrm{CdCl}_{2}$ and $\mathrm{DIBP}$ induce caspases activation}

In order to understand whether the decrease of sperm viability and motility observed after incubation with the two environmental pollutants was due to induction of an apoptotic pathway, we evaluated the percentage of spermatozoa expressing activated caspases 3 and 7, considered the main effectors of apoptosis (Grunewald et al. 2009). We found that exposure to $10 \mu \mathrm{M} \mathrm{CdCl}_{2}$ or $200 \mu \mathrm{M}$ DIBP for $24 \mathrm{~h}$ resulted in a significant increase of caspases activity (Fig. 3, $n=7$ ). 
Table 2 Median values (IQR) of kinematic sperm parameters, evaluated by CASA system, are reported in control samples and in samples exposed to $10 \mu \mathrm{M} \mathrm{CdCl}_{2}$ or $100 \mu \mathrm{M}$ DIBP or $200 \mu \mathrm{M}$ DIBP for $24 \mathrm{~h}(n=25)$.

\begin{tabular}{lccrr}
\hline CASA parameter & CTRL & $\mathbf{1 0} \boldsymbol{\mu} \mathbf{M ~ C d C l}_{\mathbf{2}}$ & $\mathbf{1 0 0} \boldsymbol{\mu M} \mathbf{D I B P}$ & $\mathbf{2 0 0} \boldsymbol{\mu M} \mathbf{D I B P}$ \\
\hline VAP $(\mu \mathrm{m} / \mathrm{s})$ & $69.2(63.7-74.4)$ & $64.6^{*}(60.3-71.6)$ & $65.0^{\S}(60.2-71.9)$ & $57.2^{\wedge^{\circ}(54.5-65.1)}$ \\
VSL $(\mu \mathrm{m} / \mathrm{s})$ & $56.4(51.5-62.7)$ & $57.5(50.8-59.5)$ & $53.7^{\S}(49.2-55.5)$ & $47.8^{\wedge}(44.4-52.5)$ \\
VCL $(\mu \mathrm{m} / \mathrm{s})$ & $118.3(104.5-129.3)$ & $111.4^{*}(99.5-117.7)$ & $108.8^{\S}(100.1-136.5)$ & $102.1^{\wedge}(91.8-123.1)$ \\
ALH $(\mu \mathrm{m})$ & $5.4(4.7-5.8)$ & $5.1(4.4-5.7)$ & $5.0(4.6-6.0)$ & $5.2(4.3-6.0)$ \\
BCF $(\mathrm{Hz})$ & $20.1(18.4-22.7)$ & $21.7(16.9-24.0)$ & $18.9(17.6-21.2)$ & $18.2^{\wedge}(17.0-18.9)$ \\
STR $(\%)$ & $84.0(80.9-86.0)$ & $86.5^{*}(82.8-89.0)$ & $81.0(77.3-85.8)$ & $82.0^{\wedge}(79.3-85.0)$ \\
LIN $(\%)$ & $50.0(47.0-53.1)$ & $52.5(49.3-57.3)$ & $48.0(43.5-53.0)$ & $47.8^{\wedge}(45.3-53.1)$ \\
HA $(\%)$ & $15.0(5.0-24.0)$ & $5.0^{*}(0.0-11.3)$ & $11.5^{\S}(4.0-27.5)$ & $6.0^{\wedge}(2.6-21.0)$ \\
\hline
\end{tabular}

Wilcoxon test: $* P<0.0510 \mu \mathrm{M} \mathrm{CdCl}_{2}$ vs CTRL; ${ }^{\circledR} P<0.05100 \mu \mathrm{M}$ DIBP vs CTRL; $\wedge P<0.05200 \mu \mathrm{M}$ DIBP vs CTRL; ${ }^{\circ} P<0.05200 \mu \mathrm{M}$ DIBP vs $100 \mu \mathrm{M}$ DIBP.

ALH, amplitude of lateral head displacement; BCF, beat cross frequency; HA, hyperactivated motility; LIN, linearity of progression; STR, straightness; VAP, average path velocity; VCL, curvilinear velocity; VSL, straight line velocity.

\section{$\mathrm{CdCl}_{2}$ and DIBP effects on sperm acrosome reaction}

Next, we evaluated the effect of the two contaminants on the ability of sperm to undergo acrosome reaction in basal conditions and in response to the steroid progesterone, considered a physiological inducer of the process (Baldi et al. 2009). As can be observed in Fig. 4, spontaneous (basal) acrosome reaction in viable spermatozoa was significantly increased after exposure to $10 \mu \mathrm{M} \mathrm{CdCl}_{2}$ and $200 \mu \mathrm{M}$ DIBP, $n=10$. As expected, in untreated samples, the percentage of acrosomereacted spermatozoa increased after stimulation with progesterone (Fig. 4). Interestingly, in $\mathrm{CdCl}_{2}$ - and DIBPtreated samples, incubation with the steroid hormone did not result in an increase of the percentage of acrosome-reacted spermatozoa compared to respective basal condition (Fig. 4). These data suggest that exposure to toxic agents may induce an early acrosome reaction of human spermatozoa blunting the physiological response to progesterone.

\section{$\mathrm{CdCl}_{2}$ and DIBP effects on sperm intracellular calcium levels}

Because the entry of calcium is involved in triggering acrosome reaction, we evaluated the intracellular

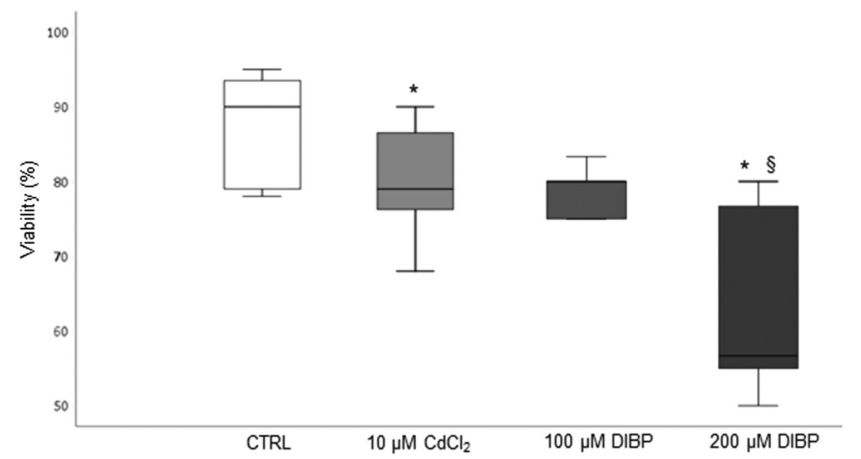

Figure 2 Box plots represent percentage of sperm viability in control samples (CTRL) and in samples exposed to $10 \mu \mathrm{M} \mathrm{CdCl}$ or $100 \mu \mathrm{M}$ DIBP or $200 \mu \mathrm{M}$ DIBP for $24 \mathrm{~h}(n=15)$. Wilcoxon test: ${ }^{*} P<0.05$ vs CTRL; ${ }^{\$} P<0.05$ vs $100 \mu \mathrm{M}$ DIBP. levels $\left(\left[\mathrm{Ca}^{2+}\right]_{\mathrm{i}}\right)$ of such ion after incubation with the two pollutants. We found that $24 \mathrm{~h}$ incubation with $\mathrm{CdCl}_{2}$ resulted in an increase in basal $\left[\mathrm{Ca}^{2+}\right]_{i}$ levels (Fig. $5 \mathrm{~A}$ and $B, n=9)$. Conversely, incubation with DIBP did not affect basal $\left[\mathrm{Ca}^{2+}\right]_{i}$ levels (Fig. 5B). After stimulation with progesterone, the expected increase of $\left[\mathrm{Ca}^{2+}\right]_{1}$ levels was observed both in untreated and treated samples.

\section{Combined effect of $\mathrm{CdCl}_{2}$ and DIBP on sperm motility}

In order to evaluate if a synergistic effect between the two toxicants may occur, spermatozoa were incubated with the mixture of both compounds for $24 \mathrm{~h}(n=8)$. On the basis of results on sperm motility and viability (Figs 1 , 2 and Supplementary Fig. 1), we chose the doses of 5 and $10 \mu \mathrm{M}$ for $\mathrm{CdCl}_{2}$ and $100 \mu \mathrm{M}$ for DIBP. As shown in Fig. 6, a significant decrease of progressive motility was observed for $10 \mu \mathrm{M} \mathrm{CdCl} 2+100 \mu \mathrm{M}$ DIBP (MIX 1) compared both to control and to single treatments, whereas no significant effect was observed for $5 \mu \mathrm{M}$ $\mathrm{CdCl}_{2}+100 \mu \mathrm{M}$ DIBP (MIX 2, not shown). MIX 1 also resulted in a significant reduction of sperm viability both compared to control and to $10 \mu \mathrm{M} \mathrm{CdCl}_{2}$ (median values: control, $87.0 \%$ (IQR: 78.5-90.0); $10 \mu \mathrm{M} \mathrm{CdCl}_{2}$,

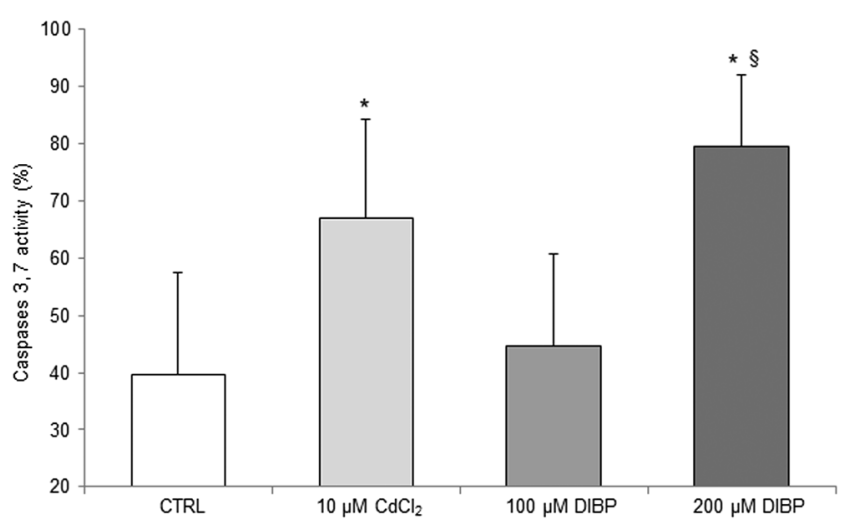

Figure 3 Histograms represent percentage of sperm caspases 3 and 7 activity in control samples (CTRL) and in samples exposed to $10 \mu \mathrm{M}$ $\mathrm{CdCl}_{2}$ or $100 \mu \mathrm{M} \mathrm{DIBP}$ or $200 \mu \mathrm{M}$ DIBP for $24 \mathrm{~h}(n=7)$. Paired $t$-test: $* P<0.05$ vs CTRL; ${ }^{\circledR} P<0.05$ vs $100 \mu \mathrm{M}$ DIBP. 


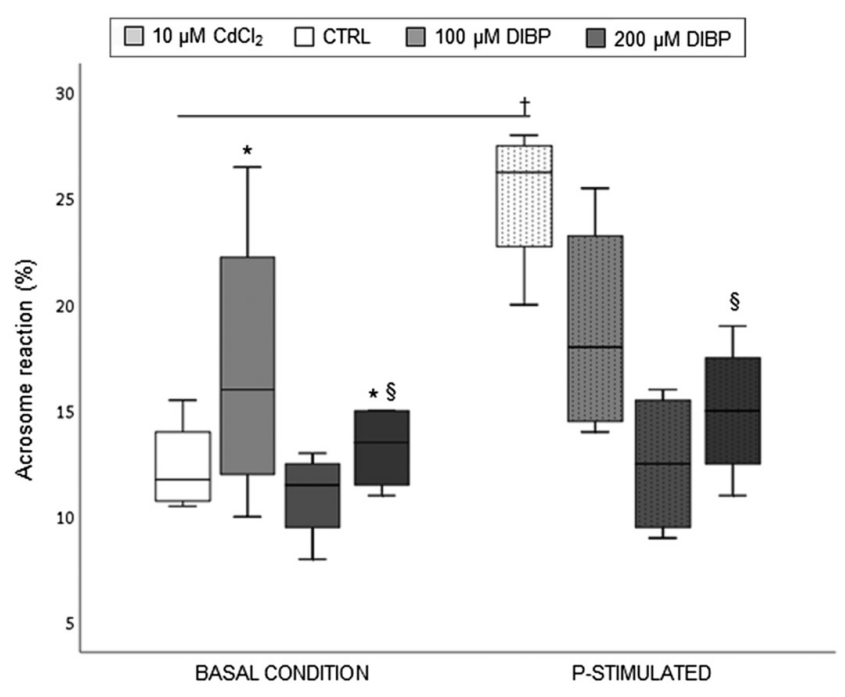

Figure 4 Box plots represent percentage of acrosome-reacted spermatozoa in basal condition (left) and after stimulation with progesterone $(\mathrm{P}$, right) in control samples (CTRL) and in samples exposed to $10 \mu \mathrm{M} \mathrm{CdCl}_{2}$ or $100 \mu \mathrm{M}$ DIBP or $200 \mu \mathrm{M}$ DIBP for $24 \mathrm{~h}$ $(n=10)$. Wilcoxon test: ${ }^{\dagger} P<0.05$, P-stimulated vs basal condition in CTRL samples; ${ }^{*} P<0.05$ vs CTRL; ${ }^{\circledR} P<0.05$ vs $100 \mu \mathrm{M}$ DIBP.

69.5\% (IQR: 63.5-87.5); MIX 1, 57.5\% (IQR: 52.364.8); $P<0.05)$. Finally, MIX 1 determined a further decrease of HA of $20.0 \%$ (IQR: 18.2-45.5) compared to $10 \mu \mathrm{M} \mathrm{CdCl}_{2}(P=\mathrm{ns})$ and of $50.0 \%$ (IQR: $\left.6.3-71.0\right)$ compared to $100 \mu \mathrm{M}$ DIBP $(P<0.05)$.

\section{Discussion}

The general population is daily exposed to several environmental contaminants by inhalation, ingestion and through the skin. The long-term exposure to environmental chemicals can lead to their accumulation in the organism, damaging organs and tissues. Reproductive systems appear to be particularly sensitive to the endocrine-disrupting action of these agents (Sifakis et al. 2017), which may provoke alterations of gonadal function and of other structures of the reproductive tracts as well as impact on gamete functions. In the present study, we demonstrate the occurrence of detrimental effects on human sperm functions necessary for fertilization after acute in vitro exposure to two toxic agents $\left(\mathrm{CdCl}_{2}\right.$ and DIBP) representative of different categories of environmental pollutants, heavy metals and phthalates. Both compounds significantly reduced the percentage of sperm progressive and hyperactivated motility and alter the ability of spermatozoa to undergo acrosome reaction in response to the physiological stimulus progesterone (Baldi et al. 2009). Overall, our results indicate that environmental toxic agents such as $\mathrm{CdCl}_{2}$ and DIBP could damage the sperm fertilization potential. Indeed, progressive and hyperactivated motility are necessary, respectively, to
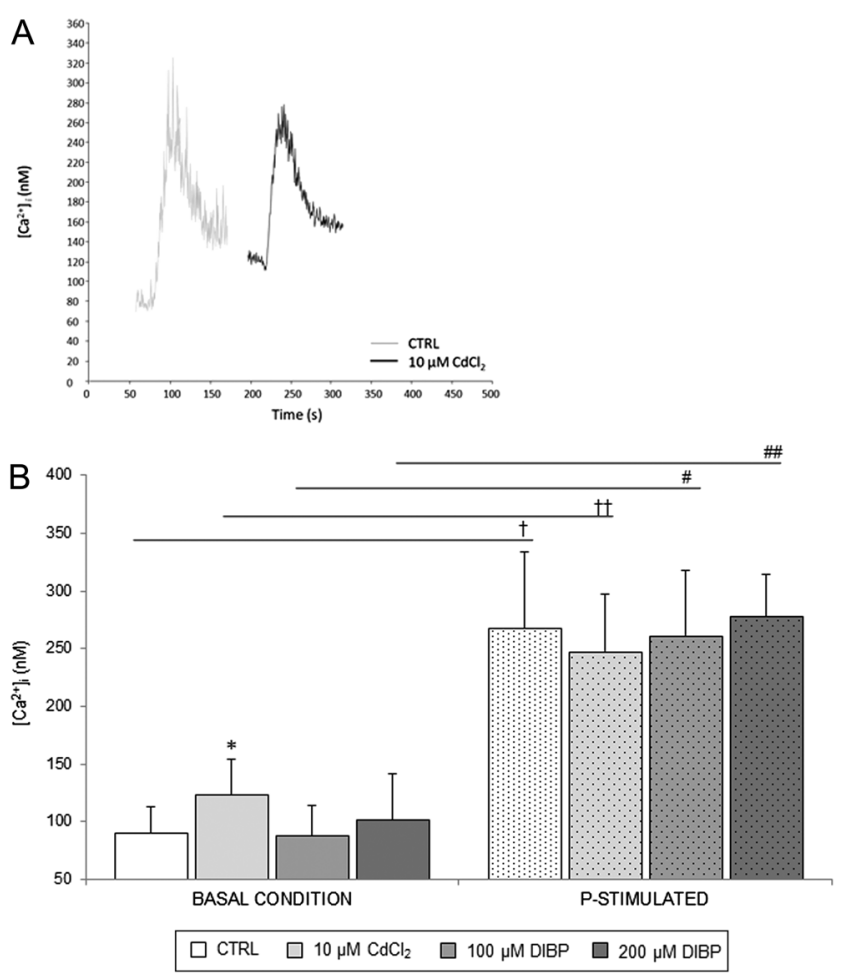

Figure 5 (A) Typical $\left[\mathrm{Ca}^{2+}\right]_{i}$ changes in response to progesterone in control samples and in samples exposed to $10 \mu \mathrm{M} \mathrm{CdCl}_{2}$. (B) Histograms represent $\left[\mathrm{Ca}^{2+}\right]_{\mathrm{i}}$ in basal condition (left) and after stimulation with progesterone ( $\mathrm{P}$, right) in control samples and in samples exposed to $10 \mu \mathrm{M} \mathrm{CdCl}_{2}$ or $100 \mu \mathrm{M}$ DIBP or $200 \mu \mathrm{M}$ DIBP for $24 \mathrm{~h}(n=9)$. Paired $t$-test: ${ }^{+} P<0.05$, P-stimulated vs basal condition in CTRL samples; ${ }^{+t} P<0.05$, P-stimulated vs basal condition in $10 \mu \mathrm{M}$ $\mathrm{CdCl}_{2}$-treated samples; ${ }^{*} P<0.05$, P-stimulated vs basal condition in $100 \mu \mathrm{M}$ DIBP samples; ${ }^{\#} P<0.05$, P-stimulated vs basal condition in $200 \mu \mathrm{M}$ DIBP samples; ${ }^{*} P<0.05$ vs CTRL.

reach the oocyte and to penetrate its vestments (Freitas et al. 2017), whereas acrosome reaction is needed to facilitate the fertilization process (Jin et al. 2011).

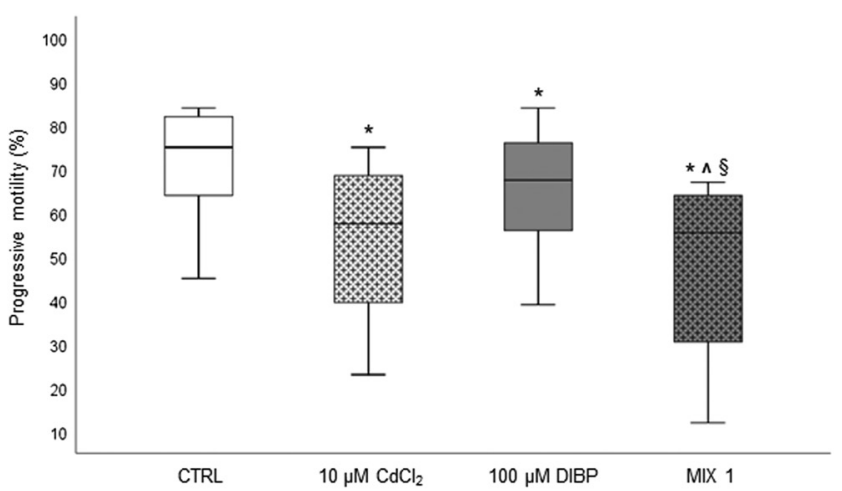

Figure 6 Box plots represent percentage of sperm progressive motility in control samples (CTRL) and in samples exposed to $10 \mu \mathrm{M} \mathrm{CdCl}_{2}$ or $100 \mu \mathrm{M}$ DIBP or MIX $1\left(10 \mu \mathrm{M} \mathrm{CdCl}_{2}+100 \mu \mathrm{M}\right.$ DIBP $)$ for $24 \mathrm{~h}(n=8)$. Wilcoxon test: $* P<0.05$ vs CTRL; $\wedge P<0.05$ vs $10 \mu \mathrm{M} \mathrm{CdCl}_{2}$; ${ } P<0.05$ vs $100 \mu \mathrm{M}$ DIBP. 
In particular, progesterone-induced acrosome reaction is highly related to fertilization ability of spermatozoa as demonstrated by numerous studies (Xu et al. 2018a). Although evaluating the effect of these compounds on the ability to fertilize of human spermatozoa is not possible for ethical reasons, it is worth mentioning that a previous study demonstrated that mouse spermatozoa exposed in vitro to cadmium produced a lower number of pronuclei with respect to controls during in vitro fertilization (Zhao et al. 2017).

A detrimental effect of cadmium on sperm motility was observed previously in other in vitro studies, where human and mouse spermatozoa were incubated with the metal at a concentration similar or higher to those used in the present study (Wang et al. 2016b, Zhao et al. 2017, Hardneck et al. 2018). A severe reduction of sperm motility and kinematic parameters were also shown in a rat model exposed to cadmium in vivo (Adamkovicova et al. 2016). Our study extends the toxic effects of the heavy metal to hyperactivated motility, which develops in the female genital tract or following in vitro incubation in capacitating media (Freitas et al. 2017). Interestingly, such effects were observed at a concentration of cadmium similar or slightly higher than those found in seminal plasma of non-professionally exposed subjects (Pant et al. 2013).

To our knowledge, no previous studies explored the in vitro effect of DIBP on spermatozoa probably because the use of this compound as plasticizer has been introduced only recently. In particular, DIBP is now used as a substitute of other phthalates, such as its isomer dibutylphthalate (DBP), because it is considered less harmful for human health (Zota et al. 2014). Our results indicate that, at high concentrations, DIBP produces a decline in the percentage of motile cells, as reported in previous studies using DBP in vitro (Pant et al. 2011, Adir et al. 2017a,b). An in vivo study on adult mice exposed to $450 \mathrm{mg} / \mathrm{kg} /$ day DIBP (Pan et al. 2017) also demonstrated a decrease of sperm motility.

The decrease of sperm viability observed in samples treated with $10 \mu \mathrm{M} \mathrm{CdCl}$ or 200 (but not 100) $\mu \mathrm{M}$ DIBP, could explain, together with the increase of the percentage of non-progressive motile spermatozoa, the observed reduction of progressive motility. Of note, the effect of the two toxicants on sperm viability does not affect the measure of hyperactivated motility and all the kinematic parameters evaluated by CASA system, because they are determined on motile spermatozoa, nor influences results of acrosome reaction, evaluated in viable spermatozoa. The decrease in sperm viability appears to be partly due to the triggering of an apoptotic cascade (Muratori et al. 2004, Said et al. 2004), as we found a significant increase of caspases 3 and 7 activity, two effectors of the apoptotic pathway, in exposed spermatozoa. Programmed cell death induction, following in vitro incubation with phthalates, has been demonstrated also in a mouse spermatocyte-derived cell line (Fu et al. 2017). We cannot exclude that the two toxicants induce an imbalance of sperm oxidative status leading to apoptosis (Bu et al. 2011, Zhou et al. 2011). Whether spermatozoa can undergo apoptosis after ejaculation is still discussed. However, recently, it has been reported that the in vitro treatment of human spermatozoa with apoptotic stimuli provokes an increase of caspase activity and typical morphological changes of apoptosis (Engel et al. 2018).

Another effect observed in spermatozoa treated with $\mathrm{CdCl}_{2}$ or $200 \mu \mathrm{M}$ DIBP is an increase of the percentage of spontaneous acrosome reaction. Since spontaneous acrosome reaction is considered a marker of acrosome stability (Xu et al. 2018b), its increase following in vitro exposure to both toxicants, could suggest an alteration of the acrosomal structure due to the two agents. It has been demonstrated, in rabbit spermatozoa, that in vitro exposure to heavy metals, including cadmium, can produce the formation of macrovesicles or large holes in the acrosome of sperm membrane (Castellini et al. 2009) and in vivo studies in mouse reported a premature sperm acrosome reaction following exposure to cadmium (Oliveira et al. 2009, Wang et al. 2017). Alterations in the acrosomal membrane could be involved also in modifying the responsiveness to progesterone. Indeed, after treatment with $\mathrm{CdCl}_{2}$ and DIBP, sperm responsiveness to progesterone was totally blunted. Although no previous studies investigated the effects of phthalates on sperm acrosome reaction, endocrine disruptors have been reported to affect such sperm function (Tavares et al. 2013, Wang et al. 2016a). At least for $\mathrm{CdCl}_{2}$, the increase of spontaneous acrosome reaction could be related also to the observed increase of basal intracellular calcium levels produced by the metal. However, since DIBP does not provoke calcium changes, such hypothesis is less plausible. Similarly, the lack of any effect of the two toxicants on $\left[\mathrm{Ca}^{2+}\right]_{\mathrm{i}}$ levels induced by progesterone suggests that other mechanisms are involved in blunting acrosome reaction in response to the steroid. It should be noted that a study evaluating concomitantly acrosome reaction and intracellular calcium increase in response to progesterone in human spermatozoa (SánchezCárdenas et al. 2014) demonstrated that the initial calcium peak in response to the steroid is not sufficient to trigger acrosomal exocytosis. Moreover, other signaling pathways are involved in acrosome reaction in response to progesterone (Baldi et al. 2009).

This study presents typical limitation of an in vitro study that cannot exactly reproduce the in vivo situation. Indeed, it is difficult to completely reflect the exposure to environmental contaminants to which the human body is subjected. However, short-term exposure (in our case $24 \mathrm{~h}$ of incubation) could represent the condition to which the spermatozoa are subjected once ejaculated and entered in the female genital tract, where they can survive for few days. It is here that, physiologically, the 
main sperm functions required for oocyte fertilization, take place. Moreover, it is unlikely that sperm cells are exposed to a single contaminant as it is more conceivable a simultaneous exposure to many toxic substances (Smarr et al. 2018). In addition, it should be considered that spermatozoa are virtually exposed to the toxicants present both in the testicular environment, seminal plasma and in the female reproductive tract, and it cannot be excluded from an additive effect. As observed in our study, incubation of spermatozoa with a mixture of $\mathrm{CdCl}_{2}$ and DIBP determined a further decrease of progressive and hyperactivated motility compared to the single exposures, suggesting that a significant interaction and a synergistic effect between the two agents may occur.

In summary, our results showed that acute in vitro exposure to $\mathrm{CdCl}_{2}$ or DIBP can compromise sperm quality and functions necessary for successful oocyte fertilization. Such findings suggest that heavy metals and phthalates might be contributing factors, among environmental pollutants, associated with progressive deterioration of male fertility potential.

\section{Supplementary data}

This is linked to the online version of the paper at https://doi.org/10.1530/REP-19-0207.

\section{Declaration of interest}

The authors declare that there is no conflict of interest that could be perceived as prejudicing the impartiality of the research reported.

\section{Funding}

This research was supported by Ministry of Education and Scientific Research (PRIN 2015 project to E Baldi, 2015XSNA83_008).

\section{Acknowledgements}

The authors also thank Drs S Dabizzi, S Degl'Innocenti and M G Fino (Azienda Ospedaliera-Universitaria Careggi) for evaluation of semen parameters.

\section{References}

Adamkovicova M, Toman R, Martiniakova M, Omelk aR, Babosova R, Krajcovicova V, Grosskopf B \& Massanyi P 2016 Sperm motility and morphology changes in rats exposed to cadmium and diazinon. Reproductive Biology and Endocrinology 14 42. (https://doi.org/10.1186/ s12958-016-0177-6)

Adir M, Salmon-Divon M, Combelles CMH, Mansur A, Cohen Y \& Machtinger R 2017a In vitro exposure of human luteinized mural granulosa cells to dibutyl phthalate affects global gene expression.
Toxicological Sciences 160 180-188. (https://doi.org/10.1093/toxsci/ $k f \times 170)$

Adir M, Combelles CMH, Mansur A, Ophir L, Hourvitz A, Orvieto R, Dor J \& Machtinger R 2017b Dibutyl phthalate impairs steroidogenesis and a subset of LH-dependent genes in cultured human mural granulosa cell in vitro. Reproductive Toxicology 69 13-18. (https://doi.org/10.1016/j. reprotox.2016.12.007)

Al-Saleh I, Coskun S, Al-Doush I, Al-Rajudi T, Al-Rouqi R, Abduljabbar M \& Al-Hassan S 2019 Exposure to phthalates in couples undergoing in vitro fertilization treatment and its association with oxidative stress and DNA damage. Environmental Research 169 396-408. (https://doi. org/10.1016/j.envres.2018.11.018)

Baldi E, Luconi M, Muratori M, Marchiani S, Tamburrino L \& Forti G 2009 Nongenomic activation of spermatozoa by steroid hormones: facts and fictions. Molecular and Cellular Endocrinology 308 39-46. (https://doi. org/10.1016/j.mce.2009.02.006)

Bonde JP, Flachs EM, Rimborg S, Glazer CH, Giwercman A, RamlauHansen CH, Hougaard KS, Høyer BB, Hærvig KK, Petersen SB et al. 2016 The epidemiologic evidence linking prenatal and postnatal exposure to endocrine disrupting chemicals with male reproductive disorders: a systematic review and meta-analysis. Human Reproduction Update 23 104-125. (https://doi.org/10.1093/humupd/dmw036)

Bu T, Mi Y, Zeng W \& Zhang C 2011 Protective effect of quercetin on cadmium-induced oxidative toxicity on germ cells in male mice. Anatomical Record 294 520-526. (https://doi.org/10.1002/ar.21317)

Castellini C, Mourvaki E, Sartini B, Cardinali R, Moretti E, Collodel G, Fortaner S, Sabbioni E \& Renieri T 2009 In vitro toxic effects of metal compounds on kinetic traits and ultrastructure of rabbit spermatozoa. Reproductive Toxicology 27 46-54. (https://doi.org/10.1016/j. reprotox.2008.12.003)

de Angelis C, Galdiero M, Pivonello C, Salzano C, Gianfrilli D, Piscitelli P, Lenzi A, Colao A \& Pivonello R 2017 The environment and male reproduction: the effect of cadmium exposure on reproductive function and its implication in fertility. Reproductive Toxicology 73 105-127. (https://doi.org/10.1016/j.reprotox.2017.07.021)

Eisenberg ML, Li S, Behr B, Cullen MR, Galusha D, Lamb DJ \& Lipshultz LI 2014 Semen quality, infertility and mortality in the USA. Human Reproduction 29 1567-1574. (https://doi.org/10.1093/humrep/deu106)

Eisenberg ML, Li S, Cullen MR \& Baker LC 2016 Increased risk of incident chronic medical conditions in infertile men: analysis of United States claims data. Fertility and Sterility 105 629-636. (https://doi.org/10.1016/j. fertnstert.2015.11.011)

Engel KM, Springsguth CH \& Grunewald S 2018 What happens to the unsuccessful spermatozoa? Andrology 6 335-344. (https://doi. org/10.1111/andr.12467)

Freitas MJ, Vijayaraghavan S \& Fardilha M 2017 Signaling mechanisms in mammalian sperm motility. Biology of Reproduction 96 2-12. (https:// doi.org/10.1095/biolreprod.116.144337)

Fu G, Dai J, Zhang D, Zhu L, Tang X, Zhang L, Zhou T, Duan P, Quan C, Zhang Z et al. 2017 Di(2-ethylhexyl) phthalate induces apoptosis through mitochondrial pathway in GC-2spd cells. Environmental Toxicology 32 1055-1064. (https://doi.org/10.1002/tox.22304)

Grunewald S, Sharma R, Paasch U, Glander HJ \& Agarwal A 2009 Impact of caspase activation in human spermatozoa. Microscopy Research and Technique 72 878-888. (https://doi.org/10.1002/jemt.20732)

Grynkiewicz G, Poenie M \& Tsien RY 1985 A new generation of Ca2+ indicators with greatly improved fluorescence properties. Journal of Biological Chemistry 260 3440-3450)

Hardneck F, Israel G, Pool E \& Maree L 2018 Quantitative assessment of heavy metal effects on sperm function using computer-aided sperm analysis and cytotoxicity assays. Andrologia 50 e13141. (https://doi. org/10.1111/and.13141)

Jensen TK, Jacobsen R, Christensen K, Nielsen NC \& Bostofte E 2009 Good semen quality and life expectancy: a cohort study of 43,277 men. American Journal of Epidemiology 170 559-565. (https://doi. org/10.1093/aje/kwp168. Erratum in: 2009. American Journal of Epidemiology 1701453.

Jin M, Fujiwara E, Kakiuchi Y, Okabe M, Satouh Y, Baba SA, Chiba K \& Hirohashi N 2011 Most fertilizing mouse spermatozoa begin their acrosome reaction before contact with the zona pellucida during in 
vitro fertilization. PNAS 108 4892-4896. (https://doi.org/10.1073/ pnas.1018202108)

Krausz C, Bonaccorsi L, Luconi M, Fuzzi B, Criscuoli L, Pellegrini S, Forti G \& Baldi E 1995 Intracellular calcium increase and acrosome reaction in response to progesterone in human spermatozoa are correlated with in-vitro fertilization. Human Reproduction 10 120-124. (https://doi. org/10.1093/humrep/10.1.120)

Krausz C, Bonaccorsi L, Maggio P, Luconi M, Criscuoli L, Fuzzi B, Pellegrini S, Forti G \& Baldi E 1996 Two functional assays of sperm responsiveness to progesterone and their predictive values in invitro fertilization. Human Reproduction 11 1661-1667. (https://doi. org/10.1093/oxfordjournals.humrep.a019466)

Latif T, Kold Jensen T, Mehlsen J, Holmboe SA, Brinth L, Pors K, Skouby SO, Jørgensen N \& Lindahl-Jacobsen R 2017 Semen quality as a predictor of subsequent morbidity: a Danish cohort study of 4,712 men with long-term follow-up. American Journal of Epidemiology 186 910-917. (https://doi.org/10.1093/aje/kwx067)

Levine $\mathrm{H}$, Jørgensen N, Martino-Andrade A, Mendiola J, Weksler-Derri D, Mindlis I, Pinotti R \& Swan SH 2017 Temporal trends in sperm count: a systematic review and meta-regression analysis. Human Reproduction Update. Review 23 646-659. (https://doi.org/10.1093/humupd/dmx022)

Luconi M, Muratori M, Forti G \& Baldi E 1999 Identification and characterization of a novel functional estrogen receptor on human sperm membrane that interferes with progesterone effects. Journal of Clinical Endocrinology and Metabolism 84 1670-1678. (https://doi.org/10.1210/ jcem.84.5.5670)

Luconi M, Carloni V, Marra F, Ferruzzi P, Forti G \& Baldi E 2004 Increased phosphorylation of AKAP by inhibition of phosphatidylinositol 3-kinase enhances human sperm motility through tail recruitment of protein kinase A. Journal of Cell Science 117 1235-1246. (https://doi. org/10.1242/jcs.00931)

Marchiani S, Tamburrino L, Olivito B, Betti L, Azzari C, Forti G, Baldi E \& Muratori M 2014 Characterization and sorting of flow cytometric populations in human semen. Andrology 2 394-401. (https://doi. org/10.1111/j.2047-2927.2014.00208.x)

Medina MF, Arrieta MC, Villafañe MN, Klyver SMR, Odstrcil IMA \& González ME 2017 Early signs of toxicity in testes and sperm of rats exposed to low cadmium doses. Toxicology and Industrial Health 33 576-587. (https://doi.org/10.1177/0748233716689524)

Mínguez-Alarcón L, Williams PL, Chiu YH, Gaskins AJ, Nassan FL, Dadd R, Petrozza J, Hauser R, Chavarro JE \& Earth Study Team 2018 Secular trends in semen parameters among men attending a fertility center between 2000 and 2017: identifying potential predictors. Environment International 121 1297-1303. (https://doi.org/10.1016/j. envint.2018.10.052)

Mortimer ST, Swan MA \& Mortimer D 1998 Effect of seminal plasma on capacitation and hyperactivation in human spermatozoa. Human Reproduction 13 2139-2146. (https://doi.org/10.1093/ humrep/13.8.2139)

Muratori M, Porazzi I, Luconi M, Marchiani S, Forti G \& Baldi E 2004 AnnexinV binding and merocyanine staining fail to detect human sperm capacitation. Journal of Andrology 25 797-810. (https://doi. org/10.1002/j.1939-4640.2004.tb02858.x)

Muratori M, Marchiani S, Tamburrino L, Tocci V, Failli P, Forti G \& Baldi E 2008 Nuclear staining identifies two populations of human sperm with different DNA fragmentation extent and relationship with semen parameters. Human Reproduction 23 1035-1043. (https://doi. org/10.1093/humrep/den058)

Oliveira H, Spanò M, Santos C \& Pereira Mde L 2009 Adverse effects of cadmium exposure on mouse sperm. Reproductive Toxicology $\mathbf{2 8}$ 550-555. (https://doi.org/10.1016/j.reprotox.2009.08.001)

Pan Y, Wang X, Yeung LWY, Sheng N, Cui Q, Cui R, Zhang H \& Dai J 2017 Dietary exposure to di-isobutyl phthalate increases urinary 5-methyl-2'deoxycytidine level and affects reproductive function in adult male mice. Journal of Environmental Sciences 61 14-23. (https://doi.org/10.1016/j. jes.2017.04.036)

Pant N, Pant A, Shukla M, Mathur N, Gupta Y \& Saxena D 2011 Environmental and experimental exposure of phthalate esters: the toxicological consequence on human sperm. Human and Experimental Toxicology 30 507-514. (https://doi.org/10.1177/0960327110374205)
Pant N, Pant AB, Chaturvedi PK, Shukla M, Mathur N, Gupta YK \& Saxena DK 2013 Semen quality of environmentally exposed human population: the toxicological consequence. Environmental Science and Pollution Research International 20 8274-8281. (https://doi.org/10.1007/ s11356-013-1813-8)

Pant N, Kumar G, Upadhyay AD, Patel DK, Gupta YK \& Chaturvedi PK 2014 Reproductive toxicity of lead, cadmium, and phthalate exposure in men. Environmental Science and Pollution Research International 21 11066-11074. (https://doi.org/10.1007/s11356-014-2986-5)

Radke EG, Braun JM, Meeker JD \& Cooper GS 2018 Phthalate exposure and male reproductive outcomes: a systematic review of the human epidemiological evidence. Environment International 121 764-793. (https://doi.org/10.1016/j.envint.2018.07.029)

Said TM, Paasch U, Glander HJ \& Agarwal A 2004 Role of caspases in male infertility. Human Reproduction Update 10 39-51. (https://doi. org/10.1093/humupd/dmh003)

Sánchez-Cárdenas C, Servín-Vences MR, José $\mathrm{O}$, Treviño CL, HernándezCruz A \& Darszon A 2014 Acrosome reaction and $\mathrm{Ca}^{2+}$ imaging in single human spermatozoa: new regulatory roles of $\left[\mathrm{Ca}^{2+}\right]$ i. Biology of Reproduction 91 67. (https://doi.org/10.1095/biolreprod.114.119768)

Sifakis S, Androutsopoulos VP, Tsatsakis AM \& Spandidos DA 2017 Human exposure to endocrine disrupting chemicals: effects on the male and female reproductive systems. Environmental Toxicology and Pharmacology 51 56-70. (https://doi.org/10.1016/j.etap.2017.02.024)

Smarr MM, Kannan K, Sun L, Honda M, Wang W, Karthikraj R, Chen Z, Weck J \& Buck Louis GM 2018 Preconception seminal plasma concentrations of endocrine disrupting chemicals in relation to semen quality parameters among male partners planning for pregnancy. Environmental Research 167 78-86. (https://doi.org/10.1016/j. envres.2018.07.004)

Stival C, Puga Molina Ldel C, Paudel B, Buffone MG, Visconti PE \& Krapf D 2016 Sperm capacitation and acrosome reaction in mammalian sperm. Advances in Anatomy, Embryology, and Cell Biology 220 93-106. (https://doi.org/10.1007/978-3-319-30567-7_5)

Sun J, Yu G, Zhang Y, Liu X, Du C, Wang L, Li Z \& Wang C 2017 Heavy metal level in human semen with different fertility: a meta-analysis. Biological Trace Element Research 176 27-36. (https://doi.org/10.1007/ s12011-016-0804-2)

Tavares RS, Mansell S, Barratt CL, Wilson SM, Publicover SJ \& RamalhoSantos J 2013 p, $p^{\prime}$-DDE activates CatSper and compromises human sperm function at environmentally relevant concentrations. Human Reproduction 28 3167-3177. (https://doi.org/10.1093/humrep/det372)

Tavares RS, Escada-Rebelo S, Correia M, Mota PC \& Ramalho-Santos J 2016 The non-genomic effects of endocrine-disrupting chemicals on mammalian sperm. Reproduction 151 R1-R13. (https://doi.org/10.1530/ REP-15-0355)

Wang C, Yang L, Wang S, Zhang Z, Yu Y, Wang M, Cromie M, Gao W \& Wang SL 2016a The classic EDCs, phthalate esters and organochlorines, in relation to abnormal sperm quality: a systematic review with meta-analysis. Scientific Reports 6 19982. (https://doi.org/10.1038/ srep19982)

Wang L, Li Y, Fu J, Zhen L, Zhao N, Yang Q, Li S \& Li X 2016b Cadmium inhibits mouse sperm motility through inducing tyrosine phosphorylation in a specific subset of proteins. Reproductive Toxicology 63 96-106. (https://doi.org/10.1016/j.reprotox.2016.05.018)

Wang HF, Chang M, Peng TT, Yang Y, Li N, Luo T, Cheng YM, Zhou MZ, Zeng XH \& Zheng LP 2017 Exposure to cadmium impairs sperm functions by reducing CatSper in mice. Cellular Physiology and Biochemistry 42 44-54. (https://doi.org/10.1159/000477113)

Wang Y, Zhu H \& Kannan K 2019 A review of biomonitoring of phthalate exposures. Toxics 7 E21. (https://doi.org/10.3390/toxics7020021)

Wdowiak A, Wdowiak E \& Bojar I 2017 Evaluation of trace metals in follicular fluid in ICSI-treated patients. Annals of Agricultural and Environmental Medicine 25 213-218. (https://doi.org/10.26444/ aaem/75422)

World Health Organization (ed.) 2010 WHO Laboratory Manual for the Examination and Processing of Human Semen, 5th ed. Cambridge, UK: Cambridge University Press.

Xu F, Guo G, Zhu W \& Fan L 2018a Human sperm acrosome function assays are predictive of fertilization rate in vitro: a retrospective cohort 
study and meta-analysis. Reproductive Biology and Endocrinology $\mathbf{1 6}$ 81. (https://doi.org/10.1186/s12958-018-0398-y)

Xu F, Zhu H, Zhu W \& Fan L $2018 b$ Human sperm acrosomal status, acrosomal responsiveness, and acrosin are predictive of the outcomes of in vitro fertilization: a prospective cohort study. Reproductive Biology 18 344-354. (https://doi.org/10.1016/j.repbio.2018.10.007)

Zhao LL, Ru YF, Liu M, Tang JN, Zheng JF, Wu B, Gu YH \& Shi HJ 2017 Reproductive effects of cadmium on sperm function and early embryonic development in vitro. PLoS ONE 12 e0186727. (https://doi.org/10.1371/ journal.pone.0186727)

Zhou D, Wang H \&ZhangJ 2011 Di-n-butyl phthalate(DBP) exposure induces oxidative stress in epididymis of adult rats. Toxicology and Industrial Health 27 65-71. (https://doi.org/10.1177/0748233710381895)
Zota AR, Calafat AM \& Woodruff TJ 2014 Temporal trends in phthalate exposures: findings from the National Health and Nutrition Examination Survey. 2001-2010. Environmental Health Perspectives 122 235-241. (https://doi.org/10.1289/ehp.1306681)

Received 8 May 2019

First decision 6 June 2019

Revised manuscript received 4 July 2019

Accepted 23 July 2019 